\title{
Realization of Polarization Control in High-Order Harmonic Generation
}

\author{
Pei-Chi Huang (), Carlos Hernández-García, Jen-Ting Huang, Po-Yao Huang, Laura Rego (), Chih-Hsuan Lu (), \\ Shang-Da Yang, Luis Plaja, Andy H. Kung, and Ming-Chang Chen ${ }^{(1)}$
}

(Invited Paper)

\begin{abstract}
The nature of high-order harmonic generation process limits the harmonics emission to linear polarization. In this paper, we review the recent progress to generate elliptically or circularly polarized high-harmonic EUV pulses. We further demonstrate how complete control of polarization state of isolated high-harmonic pulse can be realized today by noncollinear focusing of two driving pulses with identical ellipticity but counter-rotating helicity. This paper opens a path towards the study of the fastest dynamics-down to attosecond time scales-in circular dichroism of magnetic materials, chiral molecules, and electronic spin motion.
\end{abstract}

Index Terms-Optical harmonic generation, optical polarization, ultrafast optics.

\section{INTRODUCTION}

$\mathbf{U}$ LTRAFAST optical pulse generation plays an important role in pump/probe measurement for the fastest dynamics study in microscopy. Among all of nonlinear effects to generate short pulses, high-order harmonic generation (HHG) opens a

Manuscript received October 2, 2018; revised March 25, 2019; accepted March 28, 2019. Date of publication May 29, 2019; date of current version June 20, 2019. This work was supported in part by the Taiwan Ministry of Science and Technology under Grant 108-2636-M-007-001 and Grant 105-2112M-007-030-MY3, in part by the Academia Sinica, in part by the Junta de Castilla y León under Grant SA046U16, in part by the Ministerio de Economía y Competitividad under Grant FIS2016-75652-P, in part by the Leonardo Grant for Researchers and Cultural Creators, BBVA Foundation, in part by the Ministerio de Ciencia, Innovación y Universidades for a Ramón y Cajal under Grant RYC2017-22745, in part by the European Social Fund, and in part by the Ministerio de Educación, Cultura y Deporte under Grant FPU16/02591. (Corresponding authors: P. C. Huang and M. C. Chen.)

P.-C. Huang, C.-H. Lu, and A. H. Kung are with the Institute of Photonics Technologies, National Tsing Hua University, Hsinchu 30013, Taiwan, and also with the Institute of Atomic and Molecular Sciences, Academica Sinica, Taipei 10617, Taiwan (e-mail: pc_huang@gapp.nthu.edu.tw; lzch2000@ hotmail.com; akung@ee.nthu.edu.tw).

C. Hernández-García, L. Rego, and L. Plaja are with the Grupo de Investigación en Aplicaciones del Láser y Fotónica, Departamento de Física Aplicada, University of Salamanca, Salamanca E-37008, Spain (e-mail: carlosh ergar@usal.es; laura.rego@usal.es; lplaja@usal.es).

J.-T. Huang, P.-Y. Huang, and S.-D. Yang are with the Institute of Photonics Technologies, National Tsing Hua University, Hsinchu 30013, Taiwan (e-mail: micococco@yahoo.com.tw; cpandy08@hotmail.com.tw; sdyang@ ee.nthu.edu.tw).

M.-C. Chen is with the Institute of Photonics Technologies the Department of Physics, and the Frontier Research Center on Fundamental and Applied Sciences of Matters, National Tsing Hua University, Hsinchu 30013, Taiwan (e-mail: mingchang@mx.nthu.edu.tw).

Color versions of one or more of the figures in this paper are available online at http://ieeexplore.ieee.org.

Digital Object Identifier 10.1109/JSTQE.2019.2919777 path towards the production of the shortest light pulses, with durations in the order of the attosecond time scale. The generation mechanism of HHG is easily understood by the semi-classical three-step model: an electron is ionized and accelerated by an intense driving electrical field; after half-cycle of the driving pulse, the electron is pulled back, re-colliding with the parent ion [1], [2]. The kinetic energy accumulated during propagation in the continuum is released as high-energy -or high-frequencyradiation, ranging from the extreme ultraviolet (EUV) to the soft X-rays. Due to the nature of the HHG process, attosecond bursts are emitted every half cycle of the infrared (IR) laser field, resulting in the generation of an attosecond pulse train (APT) when multi-cycle driving pulses are used. This fact deprives the attosecond time scale of the HHG radiation, since the envelope width of the APT is usually in the range of several femtoseconds. Many approaches have demonstrated the production of isolated attosecond pulses (IAPs), giving access to study attosecond dynamics [3]-[6]. However, those methods are limited to linearly polarized fields. When an elliptically polarized driving laser field is applied, the electronic wavepacket is driven away from the parent ion, decreasing the probability of recombination and thus significantly dropping the harmonic yield [7]-[11]. The availability of circularly polarized or elliptically polarized attosecond pulses is particularly interesting for the study of dynamics in circular dichromic metrology of magnetic materials, chiral molecules and electronic spin of matter waves [12]-[15]. The great challenge of HHG still remains in this community: how to control the polarization state of attosecond pulses. Though large facilities, such as synchrotrons or x-ray free electron lasers (XFEL), provide alternative access to circularly polarized EUV or X-ray radiation with high brightness (by means of specially designed undulator systems), their access is limited. In addition, the shortest pulse duration reported to date in XFELs is in the order of tens of femtoseconds, which imposes a limitation for investigating fast dynamics of electron spin motion [16]-[22]. Therefore, polarization control of an attosecond pulse on table top EUV light source is still necessary. In this letter, we review the recent progress to produce elliptically or circularly polarized attosecond pulses. In particular, we show that, today, complete polarization control of HHG is available through adjusting the ellipticity of two input driving pulses with counter-rotating helicity in a non-collinear geometry. This solves the great dilemma 
(a)

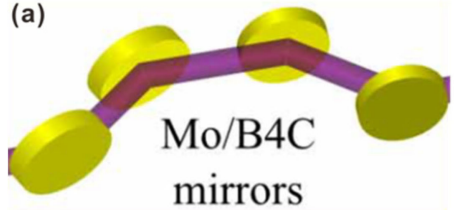

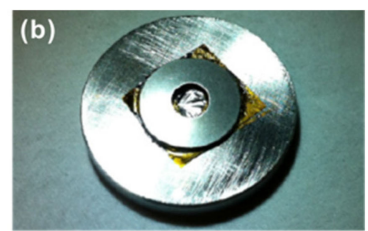

Fig. 1. (a) Multilayer coating reflective EUV retarder. The figure is reprinted with permission from ref [23], The Optical Society. (b) Multilayer coating transmission EUV retarder. The figure is reprinted with permission from ref [25], The Optical Society.

of polarization control in HHG, paving the way towards attosecond metrology in chiral systems and magnetic materials.

\section{REVIEW}

\section{A. EUV Phase Retarders}

It is hard to obtain a feasible transmission waveplate to manipulate polarization in the EUV regime due to its highly absorption nature. However, by carefully calculating the reflectivity (or transmission) and phase-shift between p- and s- polarization in material as a function of the incident angle, one can obtain an equivalent quarter waveplate in the EUV regime. B. Vodungbo et al. used four $\mathrm{Mo} / \mathrm{B}_{4} \mathrm{C}$ multilayer coating reflective mirrors [23], [24], and Schmidt et al. built a free-standing multilayer $\mathrm{Mo} / \mathrm{Si}$ foil, to generate highly elliptically polarized harmonics in the range of 46-68 eV [25], demonstrating a tabletop EUV light source with nearly pure circular polarization (see Fig. 1.). This circularly polarized EUV source is particularly interesting for spectroscopy experiments to study EUV magnetic circular dichroism (MCD) at the M-edges of Fe, $\mathrm{Co}$ and $\mathrm{Ni}$ (at 52, 60 and $67 \mathrm{eV}$, respectively). However, it suffers from poor transmission to achieve pure circular polarization as a transmission of less than $4 \%$ is reported, which especially complicates the study of time resolved $\mathrm{MCD}$, for which a huge amount of measurement time would be required.

\section{B. Elliptically Polarized Harmonics Emission by Aligned Molecules}

The efficiency of high-harmonic emission can be estimated through the product of the returning electron wave packet and the laser-free photo-recombination transition dipole in the single atom picture. In the general case, a symmetric gas target, like atomic noble elements or random molecules, is used as HHG target, resulting in the fact that the polarization of EUV is linearly polarized and parallel to that of the driving fundamental. In 2009, Zhou et al. reported elliptically polarized high harmonics emission generated from well-aligned $\mathrm{N}_{2}$ and $\mathrm{CO}_{2}$ molecules. A weak pump pulse first illuminates the target to align the molecules, and a second intense probe pulse generate high harmonics afterwards. By adjusting the orientation angle of the polarization between the pump and probe pulses, one can manipulate the amplitude, phase and orientation of the highharmonic emission, thus obtaining elliptically polarized EUV radiation. (see Fig. 2.) However, the bandwidth and polarization control $\left(\epsilon_{\mathrm{EUV}}<0.4\right)$ is limited in this scheme [26]-[28].

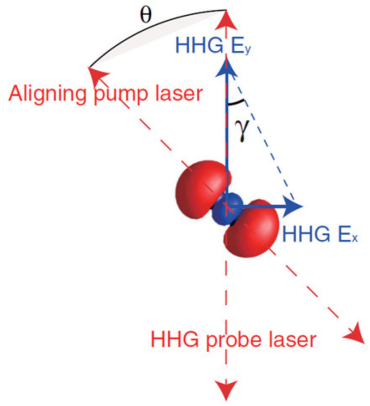

Fig. 2. HHG components parallel and perpendicular to the probe laser polarization can be generated in an aligned molecule. The figure is reprinted with permission from ref [26], American Physical Society.

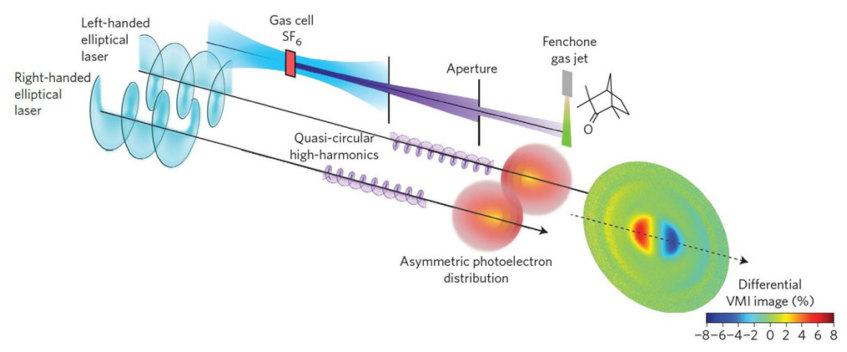

Fig. 3. Experimental set-up and principle of the PECD measurement. Leftand right-handed elliptical pulses are used alternately to generate high harmonics in $\mathrm{SF}_{6}$, and then photoionizes enantiopure fenchone molecules in a velocity map imaging spectrometer (VMIS). The photoionization of chiral molecules results in asymmetric forward/backward photoelectron emission. The figure is reprinted with permission from ref [29], Nature Publishing Group.

\section{Near Resonance HHG in Gas or Solid Targets}

In spite of the low conversion efficiency in conventional atomionized HHG, electrons in the valence band can be excited by absorbing multiple fundamental photons, and high-energy photons with polarization similar to the fundamental ones can be emitted when being released back to ground state. By this means, circularly polarized EUV can be emitted efficiently under single-color circular excitation. Ferré et al. demonstrated quasi-circularly polarized gas-targeted HHG taking advantage of resonances around the ionization threshold. They focus single circularly polarized fundamental pulses into an argon gas jet, producing harmonics with ellipticity of 0.8 at $15 \mathrm{eV}$, just below the ionization threshold of Argon. Ellipticity of less than 0.4 is found for higher-order harmonics above the ionization threshold. $\mathrm{SF}_{6}$ is also chosen used due to its multiple resonances in the range of 20 to $50 \mathrm{eV}$. The result of photoelectron circular dichroism (PECD) effect in enantiomers such as fenchone, where the emitted direction of photoelectron is related to the driving helicity, is used to prove high ellipticity $\left(\varepsilon_{E U V}>0.8\right)$ and high energy portion of polarized light [29] (see Fig. 3.). On the other hand, in solid-targeted HHG, the high-harmonic emission originates from interband and intraband mechanisms, where the first one is a result of direct eletron-hole recombination, and the later one is originated from the accelerating carrier in the band. Tancogne-Dejean et al. have demonstrated that ellipticity of the harmonics increases with increasing ellipticity of the driving field in bulk $\mathrm{MgO}$ [30], [31]. However, the HHG emission with highly elliptical polarization in solid targets is limited to 


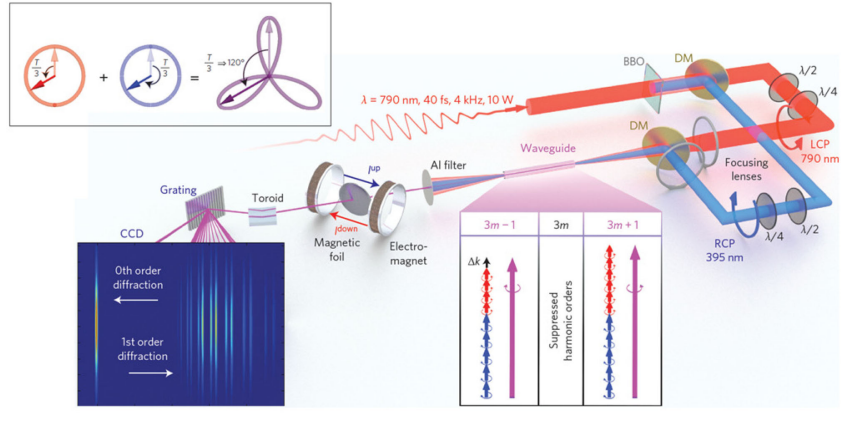

Fig. 4. Experimental apparatus of bi-color circularly polarized HHG. Right inset shows the quantum picture of generation process. Upper-left inset shows the combined electric field of a left-circularly polarized fundamental driving laser beam (red) and a counter-rotating second harmonic field (blue) is a threefold rosette shape (purple). The figure is reprinted with permission from ref [42], Nature Publishing Group.

the bandwidth nearby resonance, which is usually located at relative low photon energies. Today, it is still challenging to match the demanding bandwidth and photon energy for the study of magnetic material properties, for example, the M-edges of Fe, Co and Ni (52-67 eV).

\section{Bi-Chromatic Circularly Polarized HHG in a Collinear Geometry}

In 2008, Kitzler et al. drove HHG by fundamental field and its orthogonally polarized second harmonic (SH) field, demonstrating an increase of the up-conversion efficiency. In the meantime, through numerical simulations, they found that synthesis of the electric field not only tailors ionized electrons in time but in space, allowing electronic wavepacket recollision at different angles, so the harmonic emission can change as a function of time. As the result, an HHG spectrum with varying ellipticity for different harmonics was obtained. Theoretical prediction also shows that (1) by adjusting the frequency ratio, the ratio of peak intensity and the relative phase, polarization control can be achieved, and (2) elliptically polarized IAPs would be produced by supressing the low harmonic orders [32]. Lambert et al. experimentally realized this approach, reaching a high ellipticity, up to 0.75 , at certain harmonics orders with efficiencies similar to standard linearly polarized HHG. A MCD measurement for $\mathrm{Ni} \mathrm{M}_{2,3}$ edge around $67 \mathrm{eV}$ was demonstrated in this work [33].

In addition to the use of orthogonally linearly polarized bicolor field, counter rotating circularly polarized bi-color field was also proposed and demonstrated in the 90's [34]-[38]. Later on, in 2014, Fleischer et al. experimentally measured the polarization of the high-harmonic emission from this approach, in an experiment that supposed a breakthrough for generating high-harmonics with near pure circular polarization [39]. This bi-chromatic method consists in focusing two color circularly polarized driving pulses with counter helicity in a collinear geometry. When these two pulses overlap temporally and spatially, the combined electric field oscillates in an n-fold shape as a function of time, as shown in Fig. 4, where $n$ is determined by the least common multiple of the two frequencies. For simplifying the explanation, two pulses with wavelengths $\lambda_{1}$ and its second harmonic $\lambda_{2}=\lambda_{1} / 2$ are chosen as example in the following paragraph, in which a 3 -fold oscillation is produced. Within the semi-classical model, the electronic wavepacket experiences ionization, acceleration and recombination at each fold, emitting linearly polarized harmonics at the recombination angle of $\left(0^{\circ}, 120^{\circ},-120^{\circ}\right)$, with a time delay of $\lambda_{1} / 3 \mathrm{c}$ from each other, where $\mathrm{c}$ is the speed of light. As a result, the high-harmonic spectrum is composed by pairs of harmonics with circular polarizations but opposite helicity, obeying to the following rule: harmonic orders of $3 \mathrm{~m}-1$ present the same polarization as the $\mathrm{SH}$ field, harmonics $3 \mathrm{~m}+1$ present the polarization of the fundamental field, and harmonic orders at $3 \mathrm{~m}$, vanish, where $\mathrm{m}$ is an integer number. This can be explained from the quantum picture, through spin angular momentum conservation, which imposes $\mathrm{m}_{\lambda 1}-\mathrm{m}_{\lambda 2}= \pm 1$, where $\mathrm{m}_{\lambda 1}$ and $\mathrm{m}_{\lambda 2}$ are the number of photons absorbed from the $\lambda_{1}$ and $\lambda_{2}$ drivers (see Fig. 4) [39], [40]. Macroscopically, the phase-matching condition follows conventional single color HHG, so the critical ionized level is located between two independent linear polarized driving fields, allowing to the extend photon energies to the soft X-ray regime, for example, by using longer wavelength as driving source [41]. In this collinear scheme, the harmonic polarization is quite stable, even insensitive to the fluctuation of amplitude and phase velocity between the two colors, which is suitable for many applications, especially for the measurement of MCD of magnetic materials or chiral molecules [39]. Kfir et al. reported the MCD result of magnetized $\mathrm{Co}$ in the range of $45-60 \mathrm{eV}$ by using Ti:Sapphire amplifier at $800 \mathrm{~nm}$ and its SH at $400 \mathrm{~nm}$ [41], [42]. Fan et al. generated a broadband circularly polarized spectrum with a cutoff photon energy extending up to $160 \mathrm{eV}$ in helium gas in the use of an OPA system with two color spectra centered at 0.79 um and 1.3 um respectively. A MCD measurement of $\mathrm{Gd} \mathrm{N}_{4,5}$ edge around $145 \mathrm{eV}$ was also demonstrated [43]. This approach provides a robust solution towards circular dichroism by tabletop EUV light source without losing the up-conversion efficiency. However, the synthesis of a temporal EUV pulse with controlled polarization is limited. Pure circularly polarized pulse could be selected by use of diffractive optics to filter out specific harmonic order, but the pulse duration is limited to several femtoseconds due to the limited spectral bandwidth. Recent publications demonstrate that the temporal and polarization character of the HHG emission driven in this collinear scheme can be tailored through proper modifications of the intensity, polarization and time delay of the driving fields [44], [45]. For example, elliptical $\left(\varepsilon_{E U V} \sim 0.5\right)$ IAPs can be generated when few-cycle driving pulses are used taking advantage of the orbital symmetry at the single-atom level [45]-[47].

\section{E. Spatially Varying Ellipticity $H H G$}

More recently, Ellis et al. demonstrated HHG with spatially varying ellipticity. They first spatially separate the fundamental IR beam into two orthogonally polarized and angularly separated beams, which are then focused into an argon gas jet to independently drive two orthogonally polarized high harmonic beams. At each spatial position in the far-field, the two orthogonally polarized high harmonic beams overlap with a different time delay, thereby producing a far-field distribution with a 


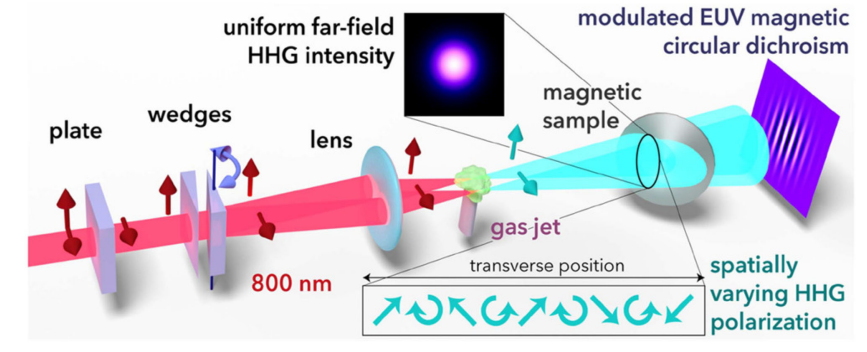

Fig. 5. Experimental setup for spatially varying ellipticity high harmonic generation (SVE-HHG). A common-path interferometer creates two orthogonally polarized and angularly separated beams, which are focused to two spatially separated focal spots for yielding two orthogonally polarized HHG beams, which diverge to overlap in the far field and thereby produce a far-field polarizationvarying distribution. The figure is reprinted with permission from ref [48], The Optical Society.

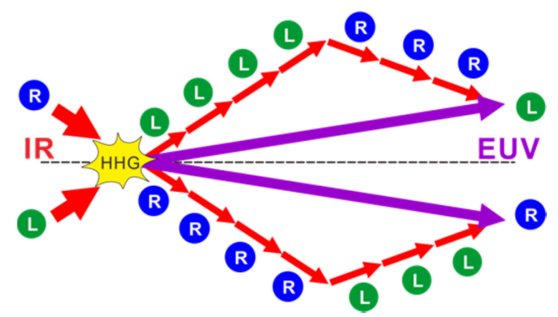

Fig. 6. Photon model of non-collinear circularly polarized high-order harmonic generation. The output direction and spin of high harmonics follow the simple linear momentum and spin angular momentum addition of absorbed fundamentals.

spatially varying ellipticity. Both linearly polarized driving lasers are independent when driving the HHG process, so they follow the single-atom and macroscopic physics as the traditional single-beam HHG, keeping the same conversion efficiency and spectral cut-off. Furthermore, it's also possible to generate IAPs by using few-cycle driving fields. EUV MCD on cobalt is measured and the result showed good spatially varying contrast of dichroism in single harmonic orders [48] (see Fig. 5.).

\section{F. Single Color Circularly Polarized HHG in Non-Collinear Geometry}

In 2015, Hickstein et al. experimentally demonstrated the generation of pure circularly polarized HHG beams by using two single-color circularly polarized drivers with opposite helicity in a non-collinear geometry [45]. While the two beams temporally and spatially overlap at focal plane, they form linearly polarized oscillations with different direction along the transverse axis. Each position can be seen as an efficient HHG emitter, and the coherent superposition in the far-field results in two counter rotating circularly polarized EUV beams. This mechanism can also be explained in the quantum picture by following the energy and spin angular momentum conservation in the non-collinearly scheme, as depicted in Fig. 6. As a result, two EUV beams, which are naturally separated from the fundamental beams, are obtained with a small crossing angle. Thus the fundamental driving beams can be removed through a spatial iris, without requiring a material filter than could cause a reduction of the HHG yield in the sample. MCD measurement at Fe sample was demonstrated in this work [45]. Although the resulting spectrum is far from the resonance of $\mathrm{Fe}$, contributing to the extremely low contrast in MCD, the experimental results reveal the robustness and stability of this approach. Different to the previous techniques, in the noncollinear geometry each EUV beam possess consistent polarization state (circular polarization here) in the full spectral range, indicating that IAPs can be achieved by using few-cycle pulse, which was revealed by numerical simulation [49], [50]. Huang et al. realized this prediction and further demonstrated that not only circular polarization, but also a complete polarization control of isolated high-harmonic pulses can be obtained in this geometry [51], [52].

Not only the polarization control of attosecond pulses, but the non-collinear HHG geometry has also been applied to the generation of circularly polarized vortices with tailored orbital angular momentum very recently [53]. It paves the way to ultrafast studies of chiral systems using high-harmonic beams with designer spin and orbital angular momentum.

\section{Complete Polarization Control of HHG}

\section{A. Theory}

To control the ellipticity of high-harmonic pulses, two identical elliptically-polarized few-cycle fundamental laser beams with ellipticity $\varepsilon_{I R}$ (equal but counter-rotating) were prepared and focused into a gas jet in a non-collinear geometry. The realization of polarization control of HHG can be obtained through this technique and it can be intuitively explained both from the quantum and wave pictures.

In the view of the quantum picture, the selection rule should be applied to the photon model. For example, in Fig. 6, when lefthanded circularly polarized fundamental goes to up to the right and right-handed circularly polarized fundamental goes to down to the right, obeying the laws of the energy, linear momentum and spin angular momentum conservation in HHG, there is only one "correct" channel (or diffraction direction) that can generate circularly polarized EUV photons.

When elliptically polarized fundamental fields are applied, one fundamental beam is made by a superposition of both the left-handed and the right-handed circularly polarized photons, wherein the superposition ratio between those two orthogonally circularly polarized photon is decided by ellipticity. When noncollinear HHG is implemented, a few possible up-conversion processes are depicted in Fig. 7 and Fig. 8, showing the accessible channels to generate counter-rotating circularly polarized high harmonic photons (corresponding to many diffracted orders in the wave pictures discussed in the following section and Fig. 9). We emphasize that EUV photons produced from the channels in Fig. 7(a) and (b) have the same output direction. Furthermore, the up-conversion probability following the channel in Fig. 7(a) is greater than that following the channel (b), resulting in elliptically polarized EUV beams through superposition as shown in Fig. 7(c), while the absorption possibility of those two orthogonally circularly polarized fundamentals determines the final helicity of EUV beams.

As the result, polarization control of HHG can be achieved by the means of adjusting the ellipticity of the fundamental 

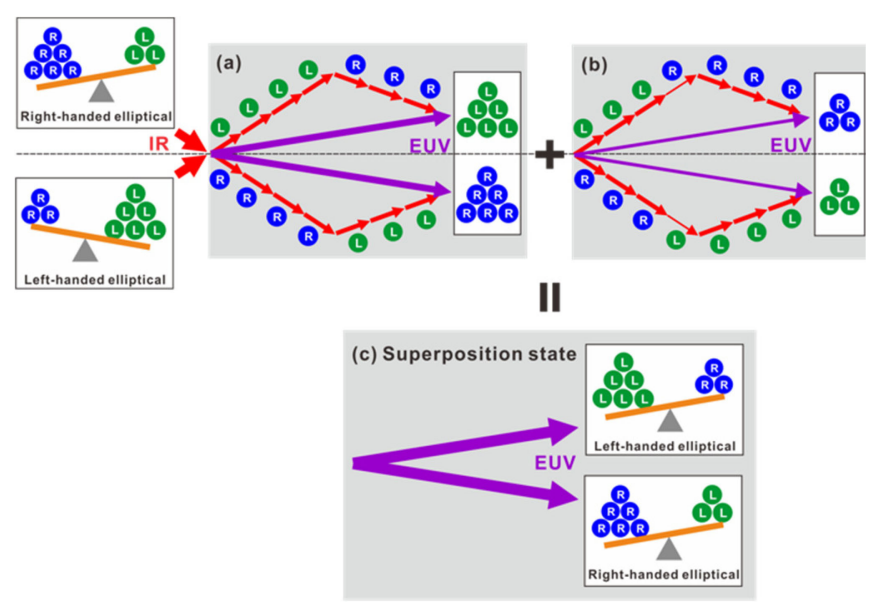

Fig. 7. Photon model of non-collinear arbitrarily polarized high harmonic generation, driven by elliptically polarized fundamentals. (a) and (b) represent two possible up-conversion channels diffracting to the same direction, where the width of the EUV arrow represents their probability. (c) shows the superposition state (elliptical polarization) of two high harmonic EUV beams.

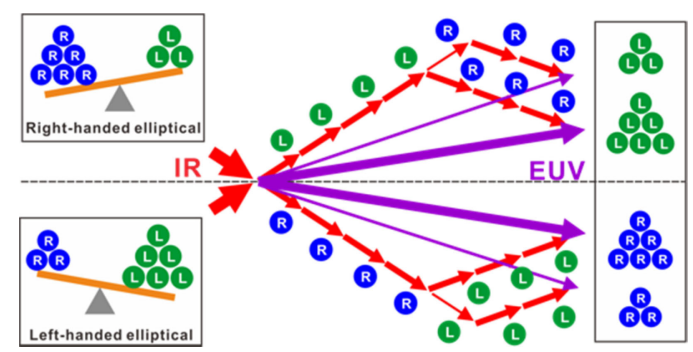

Fig. 8. Highly diffracted orders are also obeying the linear and spin angular momentum conservation laws in HHG and are elliptically polarized EUV photons.

field, i.e., changing the superposition ratio between the channel Fig. (a) and (b). Note that, in contrast to the pure-circularly polarized HHG, in which only two diffracted EUV beams appear (Fig. 6), high diffracted orders are also obeying the linear and spin angular momentum conservation law, and allowed to emit elliptically polarized EUV photons, as shown in Fig. 8.

In the view of the wave model, these two electric fields can be written as

$$
\begin{aligned}
E_{I R, R}= & \frac{\exp [i k(z \cos \theta+x \sin \theta)]}{\sqrt{1+\varepsilon_{I R}^{2}}}\left[\varepsilon_{I R} \hat{x}+i \hat{y}\right] \\
& \times \exp \left[-2 \ln 2\left(\frac{t-D}{\tau}\right)-i \omega t+i \Phi_{C E P}\right] \\
E_{I R, R}= & \frac{\exp [i k(z \cos \theta-x \sin \theta)]}{\sqrt{1+\varepsilon_{I R}^{2}}}\left[\varepsilon_{I R} \hat{x}-i \hat{y}\right] \\
& \times \exp \left[-2 \ln 2\left(\frac{t-D}{\tau}\right)-i \omega t+i \Phi_{C E P}\right]
\end{aligned}
$$

where $E_{I R, R}\left(E_{I R, L}\right)$ represents right-handed (left-handed) elliptically polarized IR light, $k=2 \pi / \lambda_{I R}$ is the fundamental wavevector, $\lambda_{I R}$ is the wavelength of the driving laser, $\theta$ is the half crossing angle between the two fundamental laser beams, $x$ and $z$ are the transverse and longitudinal distances away from the center of the focal plane respectively, $\tau$ is the full width at

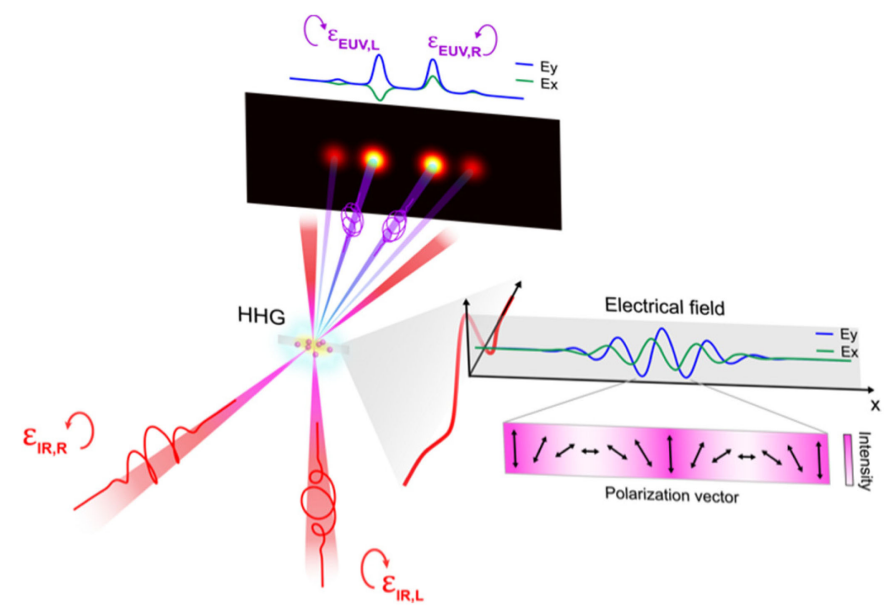

Fig. 9. Wave model of non-collinear arbitrarily polarized high harmonic generation. The up-right inset shows the electric field summed by two elliptically polarized fundamental beams on the focal plane. Summed electric field exhibits amplitude-modulated linear polarization, which rotates along the transverse direction (black arrow).

half-maximum (FWHM) pulse duration, $D$ is the delay between these two Gaussian-shaped electric fields, $\omega$ is the angular frequency of the driving laser, and $\Phi_{\mathrm{CEP}}$ is the carrier-envelope phase of driving pulses. When non-collinear HHG is implemented, the combined electric field, $E_{\text {focus }}$, at the focal plane and for perfect temporal overlap, that is $D=0$, becomes

$$
\begin{aligned}
E_{\text {focus }}(x)= & E_{I R, R}+E_{I R, L}=\frac{2}{\sqrt{1+\varepsilon_{I R}^{2}}} \\
& \times\left[\varepsilon_{I R} \cos (k x \sin \theta) \hat{x}-\sin (k x \cos \theta) \hat{y}\right] \\
& \times \exp \left[-2 \ln 2\left(\frac{t}{\tau}\right)-i \omega t+i \Phi_{C E P}\right]
\end{aligned}
$$

Therefore, E-field vectors rotating across the transverse direction $x$ with a period of $k=2 \pi /(k \sin \theta)$ are created. For any position, $x$, the local E-field oscillates linearly, which is ideal for efficient single-atom HHG. In the far-field, the high harmonic field distribution and vector varying with time can be numerically calculated through the thin slab model (TSM) [54], [55], a semiclassical method based on Fraunhofer diffraction. The diffraction integral of the spatial varying, linearly polarized oscillating high harmonic field distributed along $x$ results in two elliptically polarized or circularly polarized EUV beams. Since both amplitude and phase of HHG on the focal plane are coherently scaled and spatially imprinted by the driving IR field, the $\varepsilon_{E U V}$ can be well controlled by the $\varepsilon_{I R}$. In the case of circularly polarized harmonics, a uniform amplitude of the IR field is distributed along transverse axis on focal length, so the E-field vector from two orthogonal linearly polarized vector equally contributes and keeps a phase shift of $\lambda / 4$ to the far field, forming two circularly polarized EUV beam.

When the fundamental drivers are elliptically polarized, the IR field at focus exhibits an amplitude modulation along the transverse axis, which means that the IR amplitude of the x-polarization is different from that of the y-polarized component. Since HHG is a highly nonlinear process, the depth of 


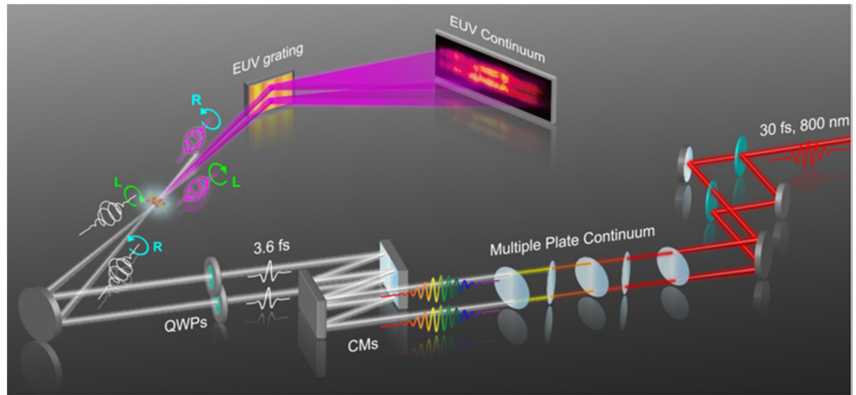

Fig. 10. Set-up for generating arbitrarily polarized isolated high-harmonic pulses using few-cycle driving lasers. CM, chirped mirror; QWP, quarter-wave plate: PM, off-axis parabolic mirror. Insets: electric field distributions at the focal plane.

resulting EUV amplitude modulation is stronger than that of fundamental due to a power-scaling rule, resulting in a lower EUV ellipticity than the input ellipticity. On the other hand, the dipole phase induced during HHG process is also related to the input intensity, so the phase shift between the two orthogonal components is no longer $\lambda / 4$. This extra phase shift originated from the dipole phase decreases the EUV ellipticity and rotates the orientation angle of the polarization ellipse of the harmonics. As a consequence of the amplitude modulation and dipole phase effect, a small variation of $\varepsilon_{I R}$ is enough to tune a wide range of $\varepsilon_{E U V}$. Moreover, the non-sinusoidal amplitude modulation spatially contributes to the arising of higher order diffraction beams, which is easily obtained by performing the spatial Fourier transform.

\section{B. Generation of Arbitrarily Polarization IAPs}

In the experiment, we start from generating two identical fewcycle IR pulses as the driving field. A supercontinuum generation performed through multiple plate continuum (MPC), is chosen, which has been reported as a robust tool to produce octave spanning spectrum ranging from $450 \mathrm{~nm}-950 \mathrm{~nm}$ in solids by using the output of Ti:sapphire amplifier [56], [57]. For the requirement of two identical duplicates of few-cycle pulses for non-collinear geometry, the unique multiplex property of MPC enable us to previously separate the output of Ti:sapphire amplifier (30 fs) by slightly misaligning a Mach-Zender interferometer with a mature optics. After simultaneously focusing two beams into an array of individual thin quartz plates to generate two almost identical supercontinuum, we compress them by the same chirped mirrors and got two identical linearly polarized fewcycle IR pulse with the pulse duration of $3.6 \mathrm{fs}$ reconstructed by polarization gating cross-correlation frequency resolved optical gating. After using two individual super-achromatic quarter waveplate (SAQWP05M-700, Thorlabs) to carefully manipulate the respective polarization to have the same ellipticity and the same orientation angle but counter-rotating helicity as mentioned in the theory section, non-collinear arbitrarily polarized HHG is implemented by focusing two beams in a crossed-beam geometry with a $17 \mathrm{mrad}$ crossing angle onto a continuous-flow argon gas jet placed in a vacuum chamber (Fig. 10).

Fig. 11. shows the resulting two EUV beam profiles from different driving ellipticities. The second-order diffraction beams
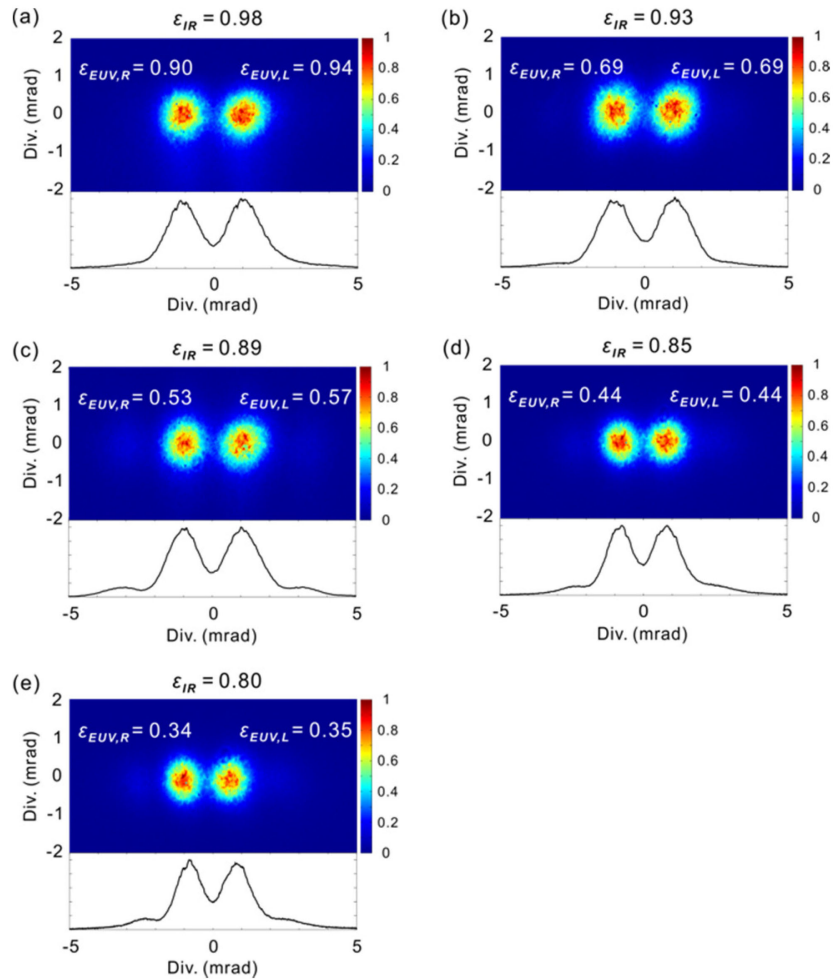

Fig. 11. The beam profile of left- and right-handed elliptically polarized highharmonic pulses with the ellipticity. (a) $\varepsilon_{E U V, R}=0.9$ and $\varepsilon_{E U V, L}=0.94$. (b) $\varepsilon_{E U V, R}=0.69$ and $\varepsilon_{E U V, L}=0.69$. (c) $\varepsilon_{E U V, R}=0.53$ and $\varepsilon_{E U V, L}=$ 0.57 . (d) $\varepsilon_{E U V, R}=0.44$ and $\varepsilon_{E U V, L}=0.44$. (e) $\varepsilon_{E U V, R}=0.34$ and $\varepsilon_{E U V, L}=0.35$

are observed besides the two first diffraction spots due to a spatially intensity modulation through the transverse direction. The induced dipole phase also significantly influences EUV ellipticity and results in the fact that EUV ellipticity drops much faster than IR ellipticity. As the result, low $\varepsilon_{E U V}$ is achieved before the intensity modulation induces strong high-order diffractions. For example, when the EUV ellipticity drops to 0.3, the intensity of the first-order diffraction pair contains $83 \%$ of the harmonic yield. Since those diffracted beams are well separated spatially, the first-order diffraction beams, can be easily selected by a simple iris for applications and analysis.

In order to study the generation of high harmonics and attosecond pulses through the noncollinear HHG scheme used in the experiment, numerical simulations are performed, including propagation using the electromagnetic field propagator [58]. The single-atom dipole acceleration is computed using the quantum strong-field approximation (SFA), without resorting in the saddle-point approximation. The signal at the detector is computed as the coherent addition of the HHG contributions of all the elementary sources, where the HHG light is assumed to propagate to the detector with a phase velocity equal to the speed of light, and propagation effects of the fundamental field are taken into account [59]. The absorption of the harmonics in the gas target is modelled using Beer's law. The temporal emission of the harmonics in the attosecond timescale is calculated by Fourier transformation of the spectral field amplitude detected at a particular far-field angle. This method has been successfully applied to the noncollinear geometry in circularly polarized 
(a)

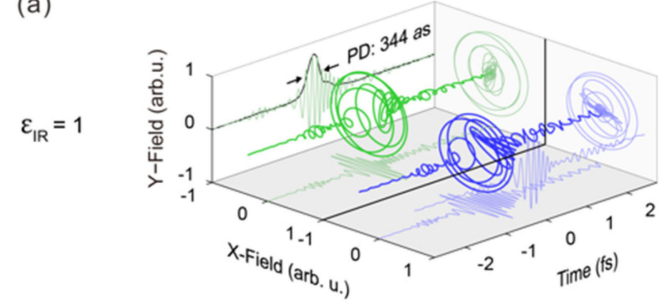

(b)

$\varepsilon_{\mathrm{IR}}=0.9$

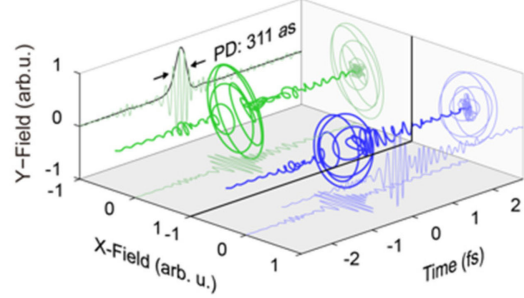

(c)

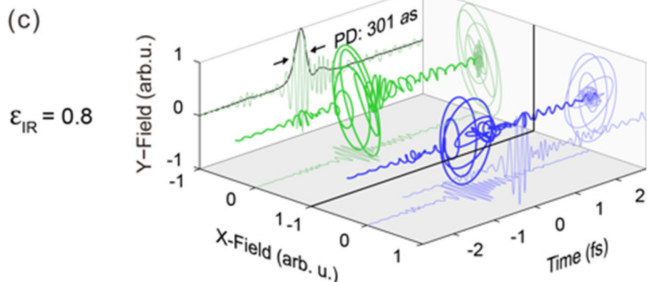

Fig. 12. Time evolution of the high-harmonic attosecond pulses obtained using $\varepsilon_{I R}=1.0(\mathrm{a}), \varepsilon_{I R}=0.9(\mathrm{~b})$ and $\varepsilon_{I R}=0.8$ (c) for the right-handed (blue) and left-handed (green) helicities detected at $\pm 1 \mathrm{mrad}$, respectively. Black lines show the temporal intensity profiles and pulse durations (PDs) of 344, 311 and 301 as, respectively.

HHG. By applying different ellipticities of the fundamental field, mimicking the experimental conditions, the simulation results show the temporal evolutions of the high-harmonic attosecond pulses obtained for (a) $\varepsilon_{I R}=1$, (b) $\varepsilon_{I R}=0.9$ and (c) $\varepsilon_{I R}=0.8$, for the right-handed (blue) and left-handed (green) helicities (see Fig. 11). As it can be observed, isolated attosecond pulses with controlled ellipticity are obtained. The duration in each case is chirped to around 300 as whereas harmonics bandwidth supports transform-limited duration of $\approx 190$ as.

Here we choose circular polarization as an experimental demonstration of isolated attosecond pulse for the reason that it is most widely used in applications. The isolation property is first characterized by grating-based EUV spectrometer, calibrated to better than $0.3 \mathrm{eV}$ resolution. To optimize the EUV continuum, the two counter-rotating driving pulses were delayed with respect to each other, to narrow the linear-polarization temporal-gating window and thus to obtain an isolated high harmonic pulse, which has been used to generate linearly polarized IAPs [5]. Fig. 13 shows the circularly polarized HHG yield and spectra as a function of a time delay between the two driving pulses. When those two driving pulses are perfectly overlapped temporally, the harmonic yield is optimized but a clear harmonic structure is visible. In our case, to generate isolated high-harmonic pulses by input pulse with duration of $3.6 \mathrm{fs}$, a time delay $\tau$ of either +1.8 or -1.8 fs (either +0.7 or -0.7 cycles) was applied to suppress temporal satellite pulses, resulting in the spectral signature of continuous spectra [50], [60],
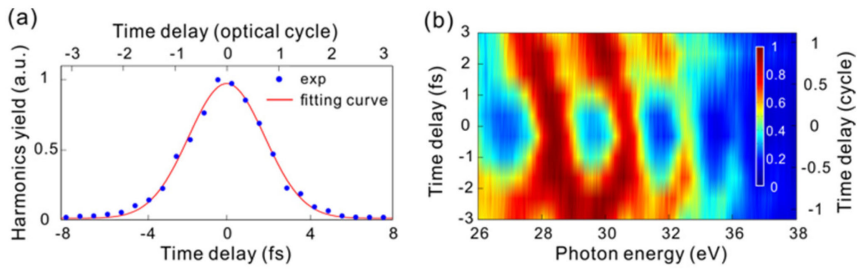

(c)
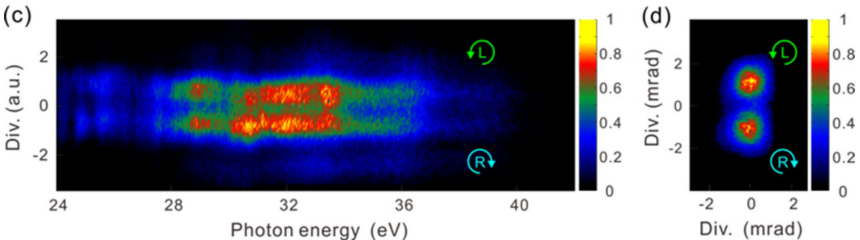

Fig. 13. (a) Measured circularly polarized harmonic yield varies with a time delay between the two driving pulses. (b) Normalized experimental circularly polarized HHG spectra as a function of a time delay, $D$, between the two driving pulses. A narrow temporal polarization gating window is created at a certain time delay, e.g., $D=+1.8 \mathrm{fs} /+0.7$ cycles or $D=-1.8 \mathrm{fs} /-0.7$ cycles, which suppresses satellite pulses, resulting in supercontinuous spectra - isolated circularly polarized high-harmonic pulses. (c) Upper and lower spectra are left-handed and right-handed circularly polarized $\mathrm{HHG}$ supercontinua with $\varepsilon_{E U V, L}=0.93$ and $\varepsilon_{E U V, R}=0.9$ and (d) their corresponding beam profiles. Normalized intensity scales are represented in the color bar.
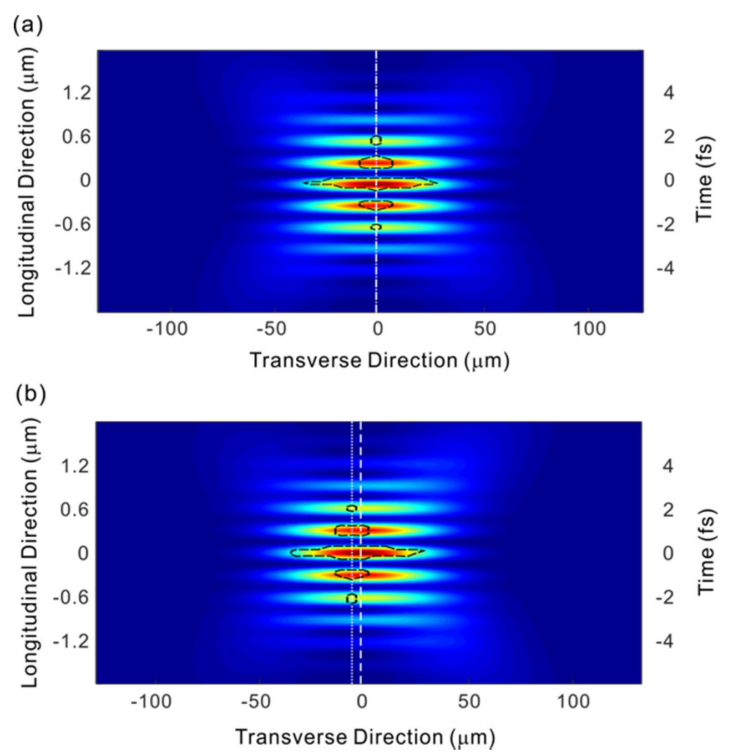

Fig. 14. 2-dimension simulation of polarization gating in the non-collinear geometry with (a) no time delay and (b) a time delay of $1.8 \mathrm{fs}$ between two drivers. The pulse duration of the two drivers is $3.6 \mathrm{fs}$. The area circled by black dashed line is the region where the polarization rotation is less than $10^{\circ}$ per femtosecond. The white dashed line indicates the position of the peak of intensity where high harmonics can be generated most efficiently, while the white dotted line indicates the position where most pulse train can be generated. Two lines are perfectly overlapped in (a), whereas the white dot-line shift to left when a time delay is applied in (b).

while sacrificing $\approx 50 \%$ high harmonics yield comparing to two driving pulses are perfectly overlapped temporally. In contrast to the conventional collinear polarization gating, a larger time delay is required in the non-collinear geometry to produce isolated attosecond pulses even using the same driving lasers. This is because the two beams in a non-collinear geometry introduce a time delay along the transverse direction [51]. Fig. 14 shows the simulation in the view of superposition of fundamental electric field at the focal plane. The horizontal axis is the transverse 
(a)

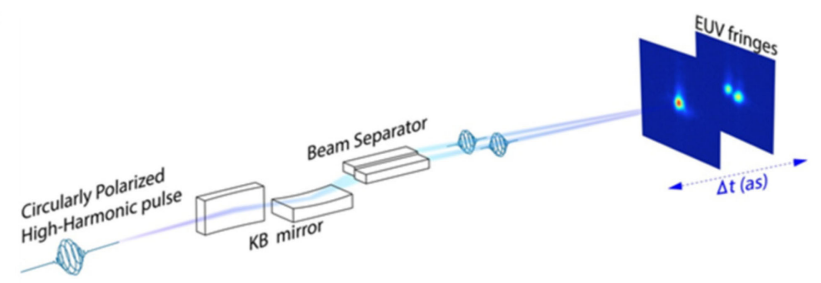

(b)

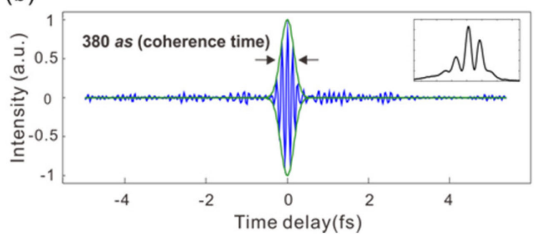

(c)

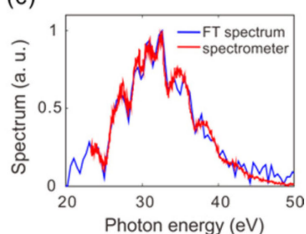

Fig. 15. (a) Scheme of the setup for EUV high-resolution Fourier transform spectroscopy. The number of discrete EUV bursts in circularly polarized HHG is directly measured by delaying one part of the HHG pulse with respect to itself with ultrahigh precision of $\sim 20$ as. (b) Experimental field autocorrelation trace of circularly polarized HHG pulses. Inset shows the visibility of spatial fringe, revealing a great coherence of circularly polarized EUV. (c) Circularly polarized EUV spectrum measured by a grating-dispersed spectrometer (red), compared with the Fourier transform of the field autocorrelation trace (blue).

position, and the vertical axis represents the propagation time. The parameters of the input pulse in the simulation are $3.6 \mathrm{fs}$, $1 \mathrm{~cm}$ separation, $20 \mathrm{~cm}$ focal length and $50 \mu \mathrm{m}$ beam size at the focal plane, which is same as the pulses used in our experiment [52]. In the case when the two circularly polarized fundamentals perfectly overlap, Fig. 14(a), the peak intensity of the beam locates at the transverse center as marked by a white dash-line. The black dashed line indicates the polarization gating window where the polarization angle rotates vs. time less than $10^{\circ}$ per femtosecond. High-harmonics exhibit high emission efficiency in this region. When a time delay is applied, the overlapping region of polarization window and high intensity region become narrow. It indicates that the generated harmonics become more isolated, which is in good agreement with the experiment. It also clearly shows that an ideal geometry for applying the polarization gating scheme in a non-collinear geometry will be a small focus spot size and a small cross angle between the two fundamentals, since it requires a lower delay between for IAPs, while keeping a high up-conversion efficiency.

To further confirm the temporal coherence of the harmonic pulses, and to distinguish the generation of an isolated EUV pulse from a train of pulses, we implement a Fourier transform field autocorrelation, as was previously done for linearly polarized pulses [6]. One of the circularly polarized EUV beams was spatially selected and sent to a split mirror, which separates the beam into two halves. A piezo actuator controls the relative time delay between the two halves of the beam with sub-nm resolution. The resulting interference pattern is measured by an X-ray $\mathrm{CCD}$ as a function of time delays, which provides the autocorrelation trace of the HHG beam. The HHG autocorrelation data (Fig. 15(b)) shows that the main EUV pulse has a high amplitude contrast ( $>10$ times) compared to adjacent pulses and a coherence length of 380 as. Fig. 15(c) shows the comparison between the spectrum extracted from a Fourier transform of the field

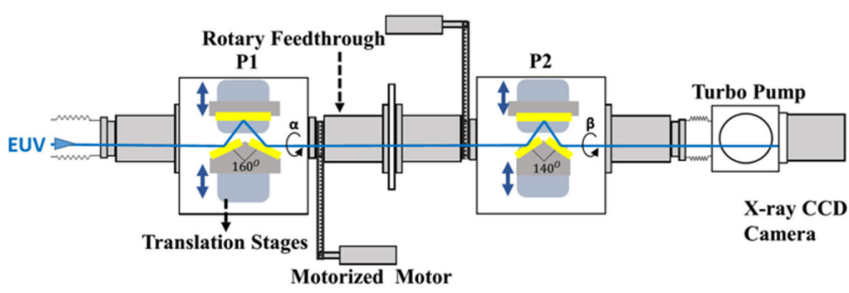

Fig. 16. Scheme of the automatic EUV polarimeter used to characterize the polarization state of isolated high-harmonic pulses, where $\alpha$ and $\beta$ are the rotating angles of the two mirror sets, respectively.

autocorrelation trace and that experimentally measured by the grating-based spectrometer. The consistency between the two spectra indicates that all circularly polarized EUV light has been successfully delivered and spatially overlapped at one focal spot, which is an essential criterion for circularly polarized attosecond pulse generation. The observed bandwidth supports transformlimited pulses duration of 190 as, while theoretical simulations predicted that isolated chirped pulses of 330 as are produced, as shown in Fig. 12.

We emphasized that though the full characterization of the three-dimensional electric field vector on attosecond timescales is necessary for clarifying the pulse broadening from attochirp in HHG process or material dispersion, it is a grand challenge to date and certainly other good research topic. For example, Jiménez-Galán et al. have already proposed a numerical solution to reconstruct the complete 3-D electric field vector from the asymmetry of the photoelectron spectra in orthogonal axis [61], [62].

\section{Complete Polarization Control and Characterization}

Due to the strong absorption in transmitting material, a device to confirm the resulting EUV polarization represents also a big challenge. Several approaches have been developed and demonstrated in synchrotron or FEL facilities. For the table-top EUV light source from HHG, a polarization analyzer by the reflectivity distinguish between s- and p- polarization on the metal coating mirror is generally used. However, it cannot recognize the portion of polarized light arising an ambiguity of the measured polarization. MCD from the property of chiral or magnetic materials provides a robust evidence by the nature of the material, especially when working for circularly polarized EUV emission. There are a few shortcomings of MCD. First, strong contrast of counter helicity appears only nearby the material resonance, which is narrow in bandwidth. Once the measured EUV spectrum is far from resonance, it requires a long acquisition time for accuracy due to the low contrast $(<1 \%)$. Second, it is hard to distinguish ellipticity and the degree of polarization when elliptical polarization is measured.

We adopt an all-optical polarimeter (Fig. 16), consisting of two independent rotatable sets of triple-reflection polarizer, where the first set acts as the phase shifter and the second set acts as a linear-polarization analyzer. Such a polarimeter unambiguously determines the ellipticity and helicity of the EUV pulses, as well as discriminating between unpolarized and polarized light by retrieving the Stokes parameters [63]. In principle, an 
(a)

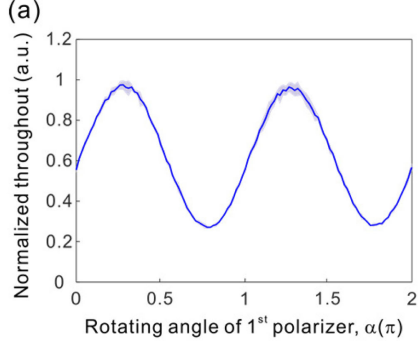

(c)

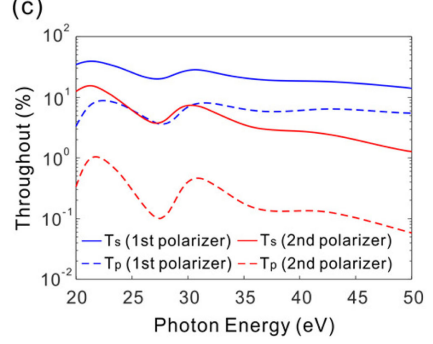

(b)

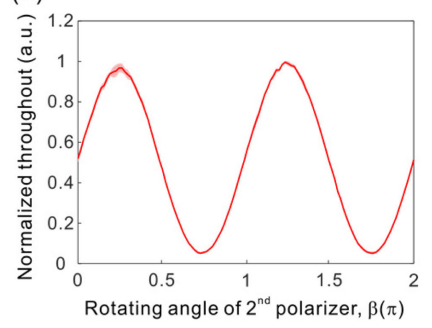

(d)

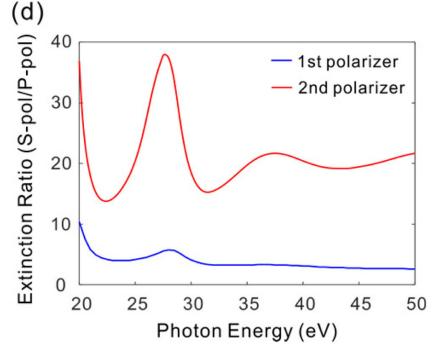

Fig. 17. (a-b) The normalized EUV throughout measured as a function of the rotating angle of the first polarizer, $\alpha$. (b) The normalized EUV throughout measured as a function of the rotating angle of the second polarizer, $\beta$. Both (a) and (b) data are averaged by 4-times measurements with its uncertainty (standard deviation) shown in light blue and red area, respectively (c) The theoretical throughput of s- and p-polarization of the two polarizers as a function of the photon energy, calculated by spectral dielectric constant of gold [64]. (d) Extinction ratios (s-polarization / p-polarization) of the two polarizers as a function of the photon energy.

all-optical polarimeter can be applied to any spectral range, if the well-design mirrors, such as metal coating mirrors or dielectric mirrors, are used in triple-reflection polarizer. In our experiment, a gold coating is chosen, which has been applied for an EUV polarimeter covering the spectral range from 5 to $80 \mathrm{eV}$.

Based on the principle of Mueller Metrix, the output intensity after passing through polarimeter is formulated as

$$
\begin{aligned}
I_{i}= & K \times F\left(S_{1} / S_{0}, S_{2} / S_{0}, S_{3} / S_{0}, \gamma_{1}, \gamma_{2}, \Delta_{1} ; \alpha, \beta\right) \\
= & K\left\{\left[1+\cos 2 \gamma_{1} \cos 2 \gamma_{2} \cos 2(\alpha-\beta)\right]\right. \\
& +S_{1} / S_{0}\left[\cos 2 \gamma_{1} \cos 2 \alpha\right. \\
& +0.5\left(1+\sin 2 \gamma_{1} \cos \Delta_{1}\right) \cos 2 \gamma_{2} \cos 2 \beta \\
& \left.+0.5\left(1-\sin 2 \gamma_{1} \cos \Delta_{1}\right) \cos 2 \gamma_{1} \cos (4 \alpha-2 \beta)\right] \\
& +S_{2} / S_{0}\left[\cos 2 \gamma_{1} \cos 2 \alpha S_{0}\right. \\
& +0.5\left(1+\sin 2 \gamma_{1} \cos \Delta_{1}\right) \cos 2 \gamma_{2} \sin 2 \beta \\
& \left.+0.5\left(1-\sin 2 \gamma_{1} \cos \Delta_{1}\right) \cos 2 \gamma_{1} \sin (4 \alpha-2 \beta)\right] \\
& +S_{3} / S_{0}\left[\sin 2 \gamma_{1} \cos 2 \gamma_{2} \sin \Delta_{1} \sin (4 \alpha-2 \beta)\right]
\end{aligned}
$$

where $K$ is a constant of the system, $S_{0}, S_{1}, S_{2}$ and $S_{3}$ are the four Stoke parameters, $r_{1}$ and $r_{2}$ are the reflectivity ratios between sand p-polarization of two mirror sets respectively, $\Delta_{1}$ is phase shift between s- and p-polarization induced by the first mirror set, $\alpha$ and $\beta$ are the respective rotating angle of the two mirror sets. In order to precisely align $\alpha$ and $\beta$ to a common horizontal,
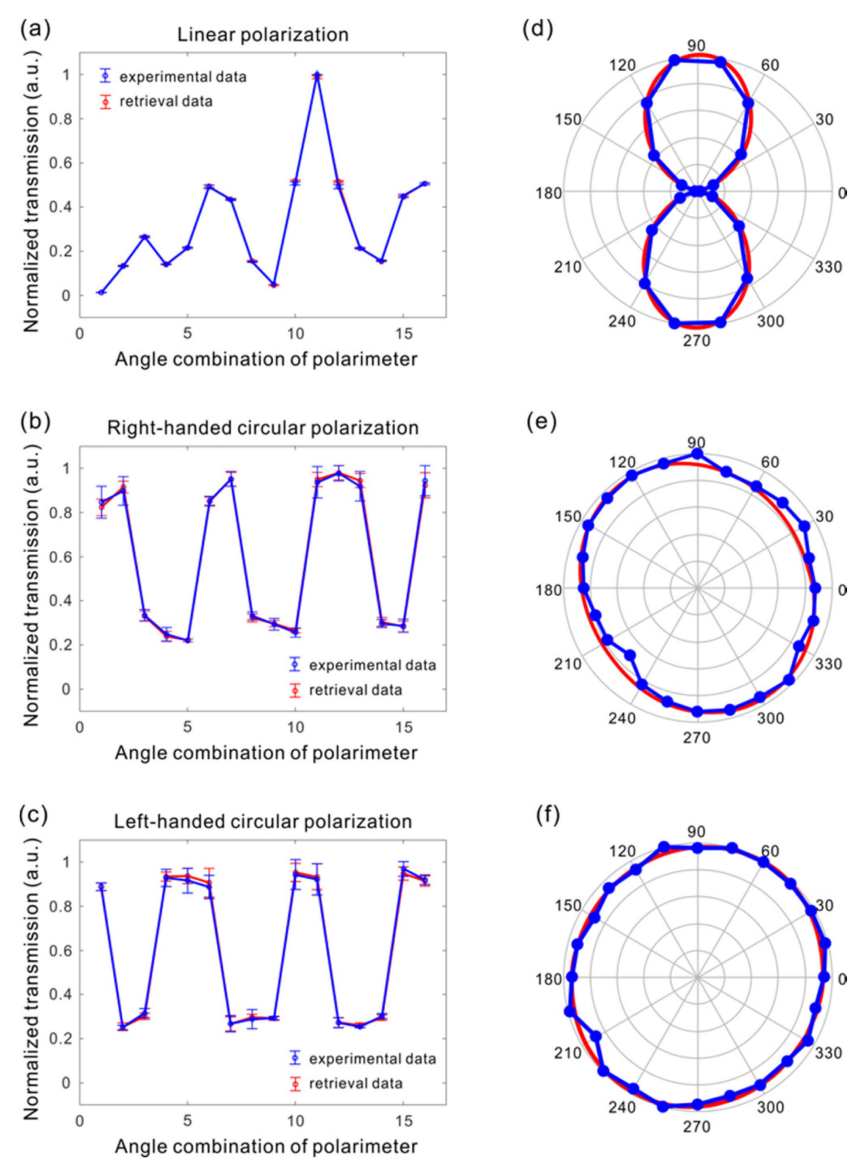

Fig. 18. (a)-(c) show the measured transmissions after all-optical polarimeter in 16 combination angles (blue) and retrieved value from the genetic algorithm (red). The significant difference between (b) and (c) represents a gooddistinguishing ability of the left-handed and right-handed circular polarization by the polarimeter. (d)-(f) corresponding angular dependent transmissions from the polarization analyzer. (a)-(d) linear polarization, (b)-(e) right-handed circular polarization, and (c)-(f) left-handed circular polarization.

a linearly polarized HHG is produced as a reference. We constructed the first polarizer and measured the linearly polarized EUV throughput as function of rotating angle $\alpha$. With keeping $\alpha$ at the angle of the maximum throughput, the second polarizer was then constructed and its throughput as the function of rotating angle $\beta$ is recorded. Figure 17(a) and (b) show the measured results. The first peak of each curve is set as the angle of zero degree of the two polarizers, respectively. The calibrations also reveal the extinction ratio of two polarizers to be 3.7 and 19 respectively, which is consistent with the theoretical value by calculating the spectral dielectric constant of gold and weighted by the spectrum in Fig. 15(c).

Although the eq. 3 provides analytical solutions to solve 7 unknown by measuring the transmissions in 7 combination angles, in order to against the power fluctuation that might be causing severe error, we took the transmissions in 16 combination angles $(\alpha, \beta)$ of $(0,0),(0, \pi / 4),(0, \pi / 2),(0,3 \pi / 4),(\pi / 4,0),(\pi / 4$, $\pi / 4),(\pi / 4, \pi / 2),(\pi / 4,3 \pi / 4),(\pi / 2,0),(\pi / 2, \pi / 4),(\pi / 2, \pi / 2),(\pi / 2$, $3 \pi / 4),(3 \pi / 4,0),(3 \pi / 4, \pi / 4),(3 \pi / 4, \pi / 2)$ and $(3 \pi / 4,3 \pi / 4)$, then we used a genetic algorithm to retrieve the Stokes parameters by minimizing the deviation of those 16 observations from their 


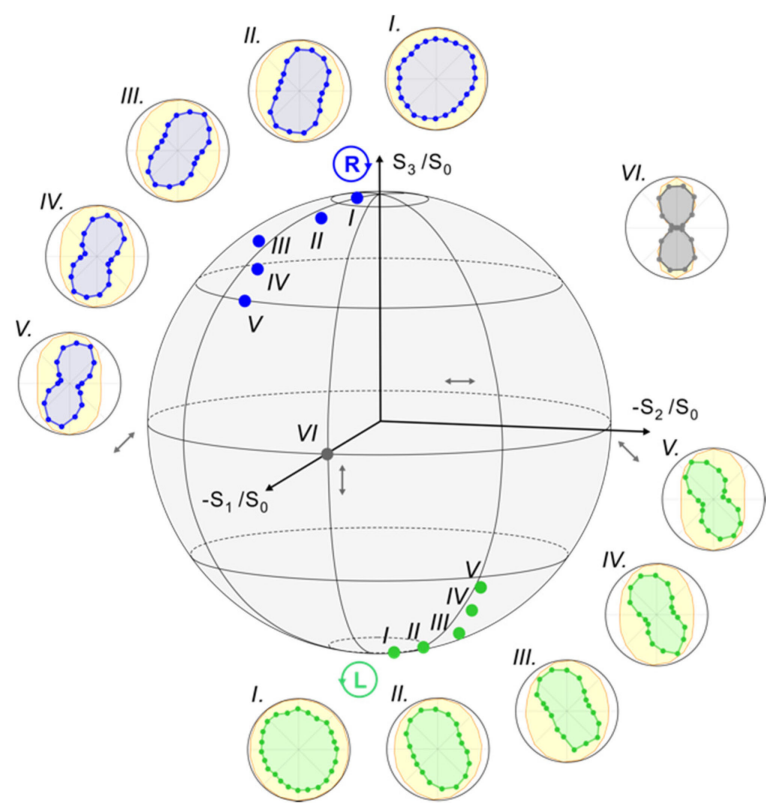

Fig. 19. Several polarization states have been generated and characterized by a polarimeter as Stokes parameters $\left(\mathrm{S}_{1} / \mathrm{S}_{0}, \mathrm{~S}_{2} / \mathrm{S}_{0}, \mathrm{~S}_{3} / \mathrm{S}_{0}\right)$, marked on the Poincaré surface. The inset polar plots show the input IR (orange lines) and output EUV (blue and green dots), measured by one IR and one EUV polarization analyzer, respectively. For comparison, linear high-harmonic pulses driven by a linearly polarized fundamental were also measured (grey dots in inset VI).

theoretical values, as shown in Fig. 16, and finally determine the polarization state.

The systematic measurement results reveal that by carefully adjusting the fundamental ellipticity from 1 to 0.8 , the EUV ellipticity can be controlled from 0.94 to 0.3 , that is, from nearly circular polarization to low elliptical polarization, as shown in Fig. 19. As observed in Fig. 12(a), when simulating the time evolution by using the same driving pulse shape as in the experiment, the polarization of the leading and tailing edge are not perfect circular polarization even though the ellipticity of input polarization is $\varepsilon_{I R}=1$. This is due to the temporal asymmetry of the input pulse shape, as shown in ref [52]. The degree of circular polarization can be further improved by applying two driving pulses with a better symmetry, as simulated by two Gaussian pulses in ref [50].

As we have theoretically predicted, the EUV ellipticity drops much faster when comparing to that of the fundamental. In this work, we obtain an ellipticity scaling rule of $\varepsilon_{E U V} \propto$ $\varepsilon_{I R}^{4.8}$, which is consistent with our numerical simulations result, $\varepsilon_{E U V} \propto \varepsilon_{I R}^{4.6}$, from the quantum and semi-classical pictures [52]. Moreover, the symmetrically tilted orientation angle of elliptical polarization is proven to result from the contribution of the dipole phase. Table I shows the detail of experimental data.

\section{SUMMARY}

In summary, we review the recent progress to produce circularly and elliptically polarized EUV light by high harmonic generation, including EUV phase retarders, harmonic emission from aligned molecules, near resonance $\mathrm{HHG}$, bi-color circularly polarized HHG in collinear geometry, and spatially varying ellipticity HHG and single color circularly polarized
TABLE I

ANALYZED ReSUlts of Polarization STATE OF ISOLATED High-HARMONIC PUlses MEASURED by AN EUV Polarimeter

\begin{tabular}{|c|c|c|c|c|c|c|}
\hline$\varepsilon_{I R, R}=\varepsilon_{I R, R}$ & 0.98 & 0.93 & 0.9 & 0.85 & 0.8 & 0 \\
\hline$\varepsilon_{E U V, R}$ & $\begin{array}{c}0.9 \pm \\
0.02\end{array}$ & $\begin{array}{c}0.69 \pm \\
0.05\end{array}$ & $\begin{array}{c}0.53 \pm \\
0.03\end{array}$ & $\begin{array}{c}0.44 \pm \\
0.04\end{array}$ & $\begin{array}{c}0.34 \pm \\
0.04\end{array}$ & $\begin{array}{c}0.006 \pm \\
0.003\end{array}$ \\
\hline $\begin{array}{c}\text { Tilt of } \\
\text { semi-major axes } \\
\psi_{E U V, R}-\psi_{I R} \\
\end{array}$ & $-29^{\circ}$ & $-16.5^{\circ}$ & $-28.6^{\circ}$ & $-19.4^{\circ}$ & $-17.5^{\circ}$ & $0^{\circ}$ \\
\hline$\varepsilon_{E U V, L}$ & $\begin{array}{c}0.94 \pm \\
0.02\end{array}$ & $\begin{array}{c}0.69 \pm \\
0.04\end{array}$ & $\begin{array}{c}0.57 \pm \\
0.04\end{array}$ & $\begin{array}{c}0.44 \pm \\
0.04\end{array}$ & $\begin{array}{c}0.35 \pm \\
0.04\end{array}$ & \\
\hline $\begin{array}{c}\text { Tilt of } \\
\text { semi-major axes } \\
\psi_{E U V, R}-\psi_{I R}\end{array}$ & $+41^{\circ}$ & $+22.3^{\circ}$ & $+27.3^{\circ}$ & $+24.2^{\circ}$ & $+23.4^{\circ}$ & \\
\hline $\begin{array}{l}\text { Harmonics yield } \\
\text { (counts per } \\
\text { second) }\end{array}$ & $3 \times 10^{7}$ & $3 \times 10^{7}$ & $2 \times 10^{7}$ & $\begin{array}{c}3.3 \\
\times 10^{7}\end{array}$ & $\begin{array}{c}3.5 \\
\times 10^{7}\end{array}$ & $5 \times 10^{7}$ \\
\hline $\begin{array}{c}\text { Polarized energy } \\
\text { portion }\end{array}$ & $85.5 \%$ & $85 \%$ & $86 \%$ & $88 \%$ & $89.5 \%$ & $99.5 \%$ \\
\hline
\end{tabular}

HHG in non-collinear geometry. We also report a full control over the polarization of HHG sources, the first arbitrarily polarized EUV source on the table-top with attosecond temporal resolution. These findings represent a new capability for the study of ultrafast dynamics in angular momentum transfer in atoms, chiral molecules, and magnetic materials, where very fast dynamics are known to occur and are not currently understood.

\section{ACKNOWLEDGMENT}

The authors would like to thank D. D. Hickstein, J. L. Ellis, Prof. C. G. Durfee, Prof. H. C. Kapteyn, and Prof. M. M. Murnane for discussion.

\section{REFERENCES}

[1] P. B. Corkum, "Plasma perspective on strong field multiphoton ionization," Phys. Rev. Lett., vol. 71, 1993, Art. no. 1994.

[2] K. J. Schafer, B. Yang, L. F. DiMauro, and K. C. Kulander, "Above threshold ionization beyond the high harmonic cutoff," Phys. Rev. Lett., vol. 70, 1993, Art. no. 1599.

[3] F. Krausz and M. Ivanov, "Attosecond physics," Rev. Mod. Phys., vol. 81, no. 163, pp. 164-234, 2009.

[4] T. Popmintchev, M.-C. Chen, P. Arpin, M. M. Murnane, and H. C. Kapteyn, "The attosecond nonlinear optics of bright coherent X-ray generation," Nature Photonics, vol. 4, pp. 822-832, 2010.

[5] I. J. Sola et al., "Controlling attosecond electron dynamics by phasestabilized polarization gating," Nature Phys. vol. 2, pp. 319-322, 2006.

[6] M.-C. Chen et al., "Generation of bright isolated attosecond soft $\mathrm{X}$-ray pulses driven by multicycle midinfrared lasers," PNAS, vol. 23, pp. E2361-E2367, 2014.

[7] P. Dietrich, N. H. Burnett, M. Ivanov, and P. B. Corkum, "High-harmonic generation and correlated two-electron multiphoton ionization with elliptically polarized light," Phys. Rev. A, vol. 50, pp. R3585-R3588, 1994.

[8] P. Antoine, A. L'Huillier, M. Lewenstein, P. Salières, and B. Carré, "Theory of high-order harmonic generation by an elliptically polarized laser field," Phys. Rev. A, vol. 53, 1996, Art. no. 1725.

[9] P. Antoine, B. Carré, A. L'Huillier, and M. Lewenstein, "Polarization of high-order harmonics," Phys. Rev. A, vol. 55, 1997, Art. no. 1314.

[10] W. Becker, A. Lohr, M. Kleber, and M. Lewenstein, "A unified theory of high-harmonic generation: Application to polarization properties of the harmonics," Phys. Rev. A, vol. 56, no. 1, pp. 645-656, 1997.

[11] S. D. Khan et al., "Ellipticity dependence of $400 \mathrm{~nm}$-driven high harmonic generation," Appl. Phys. Lett., vol. 99, 2011, Art. no. 161106.

[12] C. Boeglin et al., "Distinguishing the ultrafast dynamics of spin and orbital moments in solids," Nature, vol. 465, pp. 458-461, 2010. 
[13] J. M. N. Djiokap et al., "Electron vortices in photoionization by circularly polarized attosecond pulses," Phys. Rev. Lett., vol. 115, 2015, Art. no. 113004.

[14] R. Cireasa et al., "Probing molecular chirality on a sub-femtosecond timescale," Nature Phys., vol. 11, pp. 654-658, 2015.

[15] S. Beaulieu et al., "Probing ultrafast dynamics of chiral molecules using time-resolved photoelectron circular dichroism," Faraday Discuss., vol. 194, pp. 325-348 2016.

[16] A. A. Lutman et al., "Polarization control in an X-ray free-electron laser," Nature Photon., vol. 10, pp. 468-472, 2016.

[17] C. Spezzani et al., "Coherent light with tunable polarization from singlepass free-electron lasers," Phys. Rev. Lett., vol. 107, 2011, Art. no. 084801.

[18] D. J. Higley et al., "Femtosecond X-ray magnetic circular dichroism absorption spectroscopy at an Xray free electron laser," Rev. Scientific Instrum., vol. 87, 2016, Art. no. 033110.

[19] E. Allaria et al., "Control of the polarization of a vacuum-ultraviolet, high-gain, free-electron laser," Phys. Rev. X, vol. 4, 2014, Art. no. 041040.

[20] R. W. Schoenlein et al., "Generation of femtosecond pulses of synchrotron radiation," Science, vol. 287, no. 5461, pp. 2237-2240, 2000.

[21] S. Sasaki, K. Miyata, and T. Takada, "A new undulator for generating variably polarized radiation," Japanese J.Appl. Phys., vol. 31, pp. L1794-L1796, 1992.

[22] A. B. Temnykh, "Delta undulator for cornell energy recovery linac," Phys. Rev. Special Top.-Accel. BEAMS, vol. 11, 2008, Art. no. 120702.

[23] B. Vodungbo et al., "Polarization control of high order harmonics in the EUV photon energy range," Opt. Express, vol. 19, no. 5, pp. 4346-4356, 2011.

[24] F. Willems et al., "Probing ultrafast spin dynamics with high-harmonic magnetic circular dichroism spectroscopy," Phys. Rev. B, vol. 92, 2015, Art. no. 220405.

[25] J. Schmidt, A. Guggenmos, M. Hofstetter, S. H. Chew, and U. Kleineberg, "Generation of circularly polarized high harmonic radiation using a transmission multilayer quarter waveplate," Opt. Express, vol. 23, no. 26, pp. 33564-33578, 2015.

[26] X. Zhou et al., "Elliptically polarized high-order harmonic emission from molecules in linearly polarized laser fields," Phys. Rev. Lett., vol. 102, 2009, Art. no. 073902.

[27] A.-T. Le, R. R. Lucchese, and C. D. Lin, "Polarization and ellipticity of high-order harmonics from aligned molecules generated by linearly polarized intense laser pulses," Phys. Rev. A, vol. 82, 2010, Art. no. 023814.

[28] S. Haessler, J. Caillat, and P. Salières, "Self-probing of molecules with high harmonic generation," J. Phys. B, vol. 44, 2011, Art. no. 203001.

[29] A. Ferré etal. "A table-top ultrashort light source in the extreme ultraviolet for circular dichroism experiments," Nature Photon., vol. 9, pp. 93-98, 2015.

[30] N. Saito et al., "Observation of selection rules for circularly polarized fields in high-harmonic generation from a crystalline solid," Optica, vol. 4, no. 11, pp. 1333-1336, 2017.

[31] N. Tancogne-Dejean, O. D. Mücke, F. X. Kärtner, and A. Rubio, "Ellipticity dependence of high-harmonic generation in solids originating from coupled intraband and interband dynamics," Nature Commun., vol. 8, 2017, Art. no. 745.

[32] M. Kitzler, X. Xie, S. Roither, A. Scrinzi, and A. Baltuska, "Angular encoding in attosecond recollision," New J. Phys., vol. 10, 2008, Art. no. 025029.

[33] G. Lambert et al., "Towards enabling femtosecond helicity-dependent spectroscopy with high-harmonic sources," Nature Commun., vol. 6, 2015, Art. no. 6167.

[34] M. D. Perry and J. K. Crane, "High-order harmonic emission from mixed fields," Phys. Rev. A, vol. 48, 1993, Art. no. R4051.

[35] H. Eichmann et al., "Polarization-dependent high-order two-color mixing," Phys. Rev. A, vol. 51, 1995, Art. no. R 3414.

[36] S. Long, W. Becker, and J. K. McIver, "Model calculations of polarizationdependent two-color high-harmonic generation," Phys. Rev. A, vol. 52, pp. 2262-2278, 1995.

[37] D. B. Milošević, W. Becker, and R. Kopold, "Generation of circularly polarized high-order harmonics by two-color coplanar field mixing," Phys. Rev. A, vol. 61, 2000, Art. no. 063403.

[38] D. B. Milošević and W. Becker, "Attosecond pulse trains with unusual nonlinear polarization," Phys. Rev. A, vol. 62, 2000, Art. no. 011403(R).

[39] A. Fleischer, O. Kfir, T. Diskin, P. Sidorenko, and O. Cohen, "Spin angular momentum and tunable polarization in high-harmonic generation," Nature Photon., vol. 8, pp. 543-549, 2014.
[40] E. Pisanty, S. Sukiasyan, and M. Ivanov, "Spin conservation in high-orderharmonic generation using bicircular fields," Phys. Rev. A, vol. 90, 2014, Art. no. 043829 .

[41] O. Kfir et al., "In-line production of a bi-circular field for generation of helically polarized high-order harmonics," Appl. Phys. Lett., vol. 108, 2016, Art. no. 211106.

[42] O. Kfir et al., "Generation of bright phase-matched circularly-polarized extreme ultraviolet high harmonics," Nature Photon., vol. 9, pp. 99-105, 2015.

[43] T. Fan et al., "Bright circularly polarized soft X-ray high harmonics for X-ray magnetic circular dichroism," PNAS, vol. 112, no. 46, pp. 14206-14211, 2015.

[44] M. V. Frolov et al., "Control of harmonic generation by the time delay between two-color, bicircular few-cycle mid-IR laser pulses," Phys. Rev Lett., vol. 120, 2018, Art. no. 263203.

[45] K. M. Dorney et al., "Helicity-selective enhancement and polarization control of attosecond high harmonic waveforms driven by bichromatic circularly polarized laser fields," Phys. Rev. Lett., vol. 119, 2017, Art. no. 063201

[46] L. Medišauskas, J. Wragg, H. V. D. Hart, and M. Y. Ivanov, "Generating isolated elliptically polarized attosecond pulses using bichromatic counterrotating circularly polarized laser fields," Phys. Rev. Lett., vol. 115, 2015, Art. no. 153001

[47] D. B. Milošević, "Generation of elliptically polarized attosecond pulse trains," Opt. Lett., vol. 40, no. 10, pp. pp. 2381-2384, 2015.

[48] J. L. Ellis et al., "High harmonics with spatially varying ellipticity," $O p$ tica, vol. 5, no. 4, pp. 479-485, 2018.

[49] D. D. Hickstein et al., "Non-collinear generation of angularly isolated circularly polarized high harmonics," Nature Photon., vol. 9, pp. 743-750, 2015.

[50] C. Hernández-García et al., "Schemes for generation of isolated attosecond pulses of pure circular polarization," Phys. Rev. A, vol. 93, 2016, Art. no. 043855 .

[51] E. R. Simpson and J. Mauritsson, "Complete polarization control," Nature Photon., vol. 12, pp. 316-317, 2018.

[52] P.-C. Huang et al., "Polarization control of isolated high-harmonic pulses," Nature Photon., vol. 12, pp. 349-354, 2018.

[53] K. M. Dorney et al., "Controlling the polarization and vortex charge of attosecond high-harmonic beams via simultaneous spin-orbit momentum conservation," Nature Photon., vol. 13, pp. 123-130, 2018.

[54] C. Hernández-García, J. S. Román, L. Plaja, and A. Picón, "Quantum-path signatures in attosecond helical beams driven by optical vortices," New J. Phys., vol. 17, 2015, Art. no. 093029.

[55] L. Rego, J. S. Román, A. Picón, L. Plaja, and C. Hernández-García, "Nonperturbative twist in the generation of extreme-ultraviolet vortex beams," Phys. Rev. Lett., vol. 117, 2016, Art. no. 163202.

[56] C.-H. Lu et al., "Generation of intense supercontinuum in condensed media," Optica, vol. 1, no. 6, pp. 400-406, 2014.

[57] Y.-C. Cheng, C.-H. Lu, Y.-Y. Lin, and A. H. Kung, "Supercontinuum generation in a multi-plate medium," Opt. Express, vol. 24, no. 7, pp. 7224-7231, 2016.

[58] C. Hernández-García et al., "High-order harmonic propagation in gases within the discrete dipole approximation," Phys. Rev. A, vol. 82, 2010, Art. no. 033432.

[59] C. Hernández-García et al., "Group velocity matching in high-order harmonic generation driven by mid-infrared lasers," New J. Phys., vol. 18, 2016, Art. no. 073031.

[60] S. N. B. C M Heyl, S. Carlström, J. Mauritsson, C. L. Arnold and A. L'Huillier, "Noncollinear optical gating," New J. Phys., vol. 16, 2014, Art. no. 052001.

[61] Á. Jiménez-Galán et al., "Attosecond recorder of the polarization state of light," Nature Commun., vol. 9, 2018, Art. no. 850.

[62] C. Chen et al., "Tomographic reconstruction of circularly polarized high-harmonic fields: 3D attosecond metrology," Sci. Adv., vol. 2, 2016, Art. no. e1501333.

[63] T. Koide et al., "Elliptical-polarization analyses of synchrotron radiation in the 5-80-eV region with a reflection polarimeter," Nuclear Instrum. Methods Phys. Res. Sect. A, vol. 308, no. 3, pp. 635-644, 1991

[64] W. S. M. Werner, K. Glantschnlg, and C. Ambrosch-Draxl, "Optical constants and inelastic electron-scattering data for 17 elemental metals," $J$. Phys. Chem. Ref. Data, vol. 38, pp. 1013-1092, 2009. 


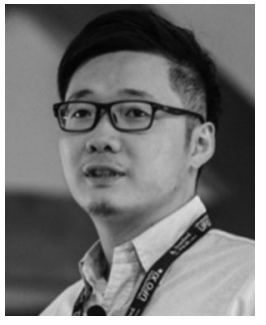

Pei-Chi Huang received the B.S. degree in electrical engineering, the M.S. and the Ph.D. degrees in electro-optical engineering from National Tsing Hua University, Hsinchu, Taiwan, in 2010, 2013 , and 2019, respectively. His research interests include generation and characterization of isolated attosecond pulses driven by few-cycle-duration fundamental fields, and complete polarization control of isolated high-harmonic pulses.

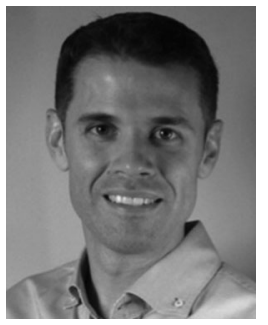

Carlos Hernández-García received the Ph.D. degree in physics from the University of Salamanca, Salamanca, Spain, in 2013. He is an Assistant Professor with the University of Salamanca. He worked for two years as Research Associate at Joint Institute for Laboratory Astrophysics, University of Colorado at Boulder, USA, within a European Marie Curie International Outgoing Fellowship. His research interests include laser-matter interaction, ultrafast phenomena, high-order harmonic generation and propagation, attosecond and zeptosecond physics, and the generation of structured light with tailored angular momentum properties in the extremeultraviolet and $\mathrm{x}$-ray regimes.

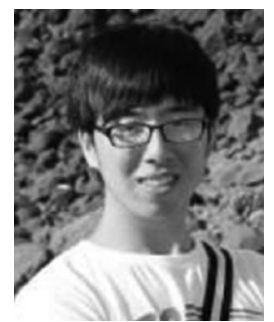

Jen-Ting Huang received the B.S. degree in electrical engineering and the M.S. degree in electro-optical engineering from National Tsing Hua University, Hsinchu, Taiwan, in 2015 and 2017. He investigated the generation of isolated circularly polarized high-harmonic pulse as topic of B.S. degree. $\mathrm{He}$ is currently employed by Artilux, Inc. in Taiwan.

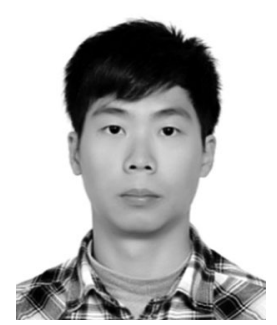

Po-Yao Huang recieved the B.S. degree in electrical engineering and life science from National Tsing Hua University, Hsinchu, Taiwan, in 2014. Since 2015, he has been working toward the M.S. degree with $\mathrm{Na}$ tional Tsing Hua University. He is currently investigating the generation of bright coherent extremeultrviolet light by using high harmonic generation and devoting himself to the characterization of EUV attosecond pulses.

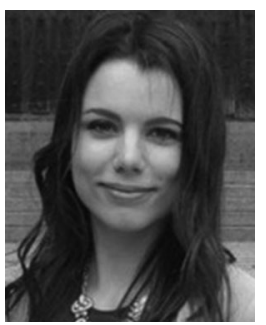

Laura Rego received the degree in physics and the master's degree from the University of Salamanca, Salamanca, Spain, in 2016 and 2017, respectively. Since 2017, she has been working toward the Ph.D. degree with the University of Salamanca. She is currently investigating new scenarios for the generation of coherent high-frequency radiation: exploring new manners of controlling the properties of extremeultraviolet/X-ray radiation, such as polarization or topological charge, and studying its generation in more complex systems, such as molecules.

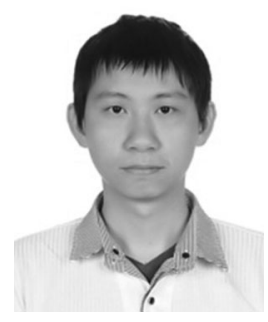

Chih-Hsuan Lu received the B.S. degree in physics from Tamkang University, New Taipei City, Taiwan, the M.S. degree in photonic from Tatung University, Taipei, Taiwan, and the Ph.D. degree from National Tsing Hua University, Hsinchu, Taiwan. He is currently a Postdoctoral with the Institute of Photonics Technologies and Electrical Engineering, National Tsing Hua University. His research focuses on the use of multiple plate continuum method for supercontinuum generation and near-single-cycle pulse compression.

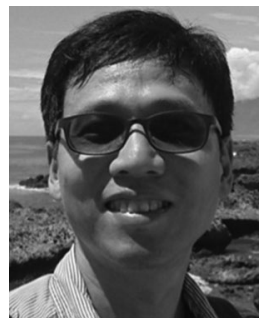

Shang-Da Yang (S'01-M'05) was born in Chiayi, Taiwan, in 1975. He received the B.S. degree in electrical engineering from National Tsing-Hua University, Hsinchu, Taiwan, in 1997, the M.S. degree in electro-optical engineering from National Taiwan University, Taipei, Taiwan, in 1999, and the Ph.D. degree from the School of Electrical and Computer Engineering, Purdue University, West Lafayette, IN USA, in 2005. He was with the Department of Electrical Engineering, National Tsing Hua University, Hsinchu, Taiwan, in 2005, and is currently a Professor. He received the RiTEK Young Investigator Medal of the Optical Engineering Society of the republic of China in 2007. He was a Visiting Professor with the Joint Institute for Laboratory Astrophysics, University of Colorado at Boulder in 2011. His research interests include characterization and synthesis of full-vectorial femtosecond waveforms, quasi-phase matching engineering, and femtosecond fiber oscillators.

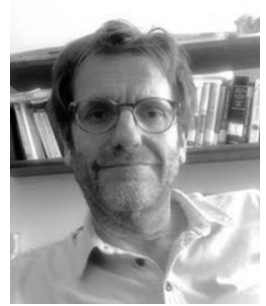

Luis Plaja received the Ph.D. degree in physics from the Universitat Autònoma de Barcelona, Bellaterra, Spain, in 1993. He is a Full Professor with the Department of Applied Physics, Universidad de Salamanca. His research interests include theoretical developments in strong field phenomena, including high harmonic generation.

Andy H. Kung currently holds the position of honorary Chair Professor with the Department of Electrical and Electronics, the National Tsing Hua University, Hsinchu, Taiwan and is a Visiting Distinguished Chair Professor with the Physics Department, Fudan University, Shanghai, China. His research interests include nonlinear optics, laser physics, and ultrafast photonics.

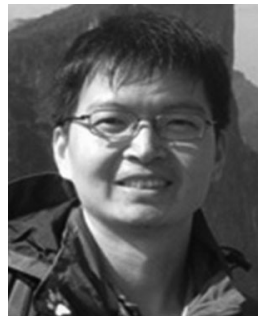

Ming-Chang Chen received the B.S. degree from National Tsing Hua University (NTHU), Hsinchu, Taiwan, the M.S. degree from the National Chiao Tung University Guangfu Campus, Hsinchu, Taiwan, and the Ph.D. degree in physics from the Joint Institute for Laboratory Astrophysics, the University of Colorado at Boulder, Boulder, Colorado, USA, in 2012. He is an Associate Professor with the Institute of Photonics Technologies and Electrical Engineering, NTHU. His research interests include demonstrating novel techniques for generation and characterization of short-duration light pulses in the deep-ultraviolet and soft X-ray regions of the spectrum, and its applications in measuring quantum dynamics in atom, molecules, and novel materials. 